\title{
Asistente computarizado para la determinación de la regla del fuera de lugar en el fútbol soccer
}

\author{
Jesús Jaime Moreno Escobar, Joshua Romero-Tapia, \\ Oswaldo Morales Matamoros \\ Instituto Politécnico Nacional, ESIME-Zacatenco, \\ Ciudad de México, México \\ jemoreno@esimez.mx
}

\begin{abstract}
Resumen. En el presente documento se presenta una metodología con el objetivo de crear un asistente para la determinación del fuera de juego utilizando visión por computadora a partir de procesos de segmentación y detección de objetos esto a partir de imágenes capturadas mediante el uso de una cámara web la cual se encontrara dentro de un espacio de iluminación controlado. Obteniendo así una imagen del terreno de juego en la cual se determinaran los elementos que estén en él, así como las distancias que ocupan dentro de la imagen para con ello obtener la existencia del fuera de juego. Para que esta metodología sea posible es necesario conocer los elementos teóricos necesarios para determinar el fuera de juego en el fútbol soccer, así como los principios de visión por computadora necesarios para realizar la captura de imágenes, la segmentación y la detección de objetos así como las herramientas necesarias para su aplicación.
\end{abstract}

Palabras clave: visión por computadora, histograma de gradiente orientado, segmentación, detección de objetos, captura de imágenes.

\section{Computerized Assistant Referee for Determining the Offside Rule in Soccer}

\begin{abstract}
In this document we present a methodology in order to create an assistant for determining the offside using computer vision from processes of segmentation and object detection this from captured using a webcam pictures which was within a space of controlled lighting. Thus, obtaining an image of the field in which the elements that are in it, and the distances they occupy within the image to thereby obtain the existence of offside is determined. For this approach possible is necessary to know the theoretical elements necessary to determine the offside-rule in soccer, as well as the principles of computer vision necessary to perform image capture, segmentation and object detection as well as the tools necessary for its implementation.
\end{abstract}

Keywords: computer vision, histogram of oriented gradient, segmentation, object detection, image capture. 


\section{Introducción}

El fútbol es uno de los deportes más populares alrededor del mundo. A lo largo de su historia siempre ha existido polémica alrededor de este deporte. Sobre todo, a la falta de exactitud de los jueces o árbitros, quienes determinan las faltas o infracciones que se dan a lo largo de un partido.

La evolución de este deporte ha provocado que el futbolista sea considerado como una inversión en comparación con épocas pasadas donde se consideraba el aspecto deportivo para el desarrollo de este deporte. Esto ha provocado un incremento en la velocidad de juego, trayendo como consecuencia la determinación de sanciones erróneas por parte de los jueces o árbitros. Sin embargo, las transmisiones televisivas son capaces de demostrar cómo estas determinaciones erróneas perjudican a alguno de los equipos que se encuentran en el terreno de juego[2].

Al presentarse con mayor frecuencia este tipo de determinaciones, en la actualidad existe un gran cuestionamiento sobre la honestidad del deporte, con lo que los organismos reguladores del fútbol buscan con mayor rapidez opciones que sean capaces de minimizar el error mediante el uso de la tecnología. Así, que mientras en deportes como el fútbol americano o el tenis existen apoyos tecnológicos capaces de ayudar a los jueces o árbitros en aquellas jugadas difíciles de analizar, en el fútbol es muy escaso el apoyo de la tecnología al existir sólo un sistema, el cual es capaz de determinar si el balón ha entrado o no en la portería rival.

Uno de estos cuestionamientos se da en la marcación del fuera de juego, la cual es decisiva en los partidos de fútbol por lo que se ha convertido en una necesidad primordial el crear un dispositivo que sea capaz de asistir en la verificación de la también conocida regla 11 del fútbol, como la menciona el reglamento de juego de la FIFA, véase la Figura 1.

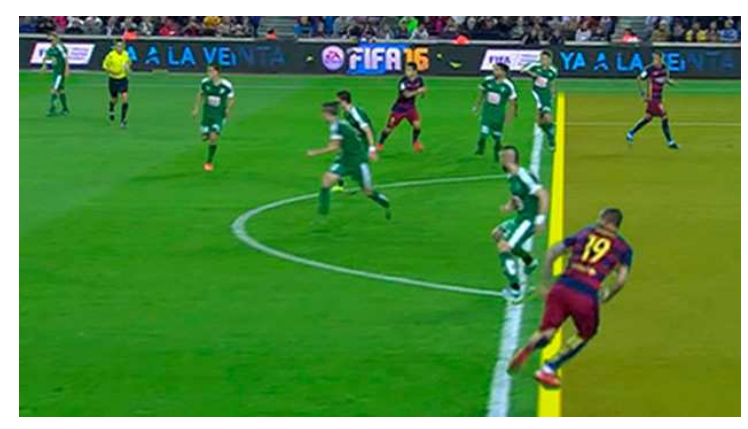

Fig. 1. Ejemplo de marcación de fuera de juego.

Establecida por la Universidad de Cambridge, en Inglaterra, en lo que se considera el primer reglamento de fútbol en el año de 1863 y modificada por la Federación Internacional de Fútbol Asociación (FIFA), en 1990. La regla del fuera de juego establece que un jugador se encontrará en posición antirreglamen- 
taria si se encuentra más cerca de la línea de meta contraria que el balón y el penúltimo adversario. Esto se toma en cuenta a partir del momento en que el balón es tocado o jugado por uno de sus compañeros, véase la Figura 2.
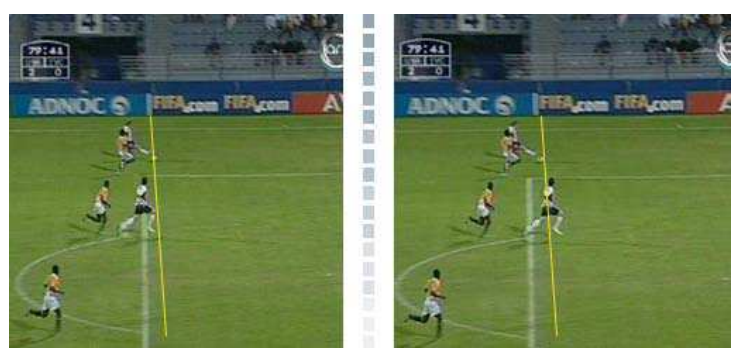

Fig. 2. Ejemplo de posición de fuera de juego [2].

Considerando que hasta el momento no existen opciones que mediante el uso de la tecnología apoye al árbitro en la determinación de esta regla, este trabajo tiene como objetivo desarrollar un asistente computacional para la determinación de la regla del fuera de juego en el fútbol soccer. Este trabajo se apoya en la visión por computadora mediante el uso de software capaz de realizar procesos de segmentación, detección de objetos y procesamiento digital de imágenes[3].

El asistente presentado en este trabajo representa una opción para apoyar a los árbitros en la toma de decisiones; esto a través de una mayor precisión en el análisis de esta regla mediante la detección de formas y sus posiciones dentro de la imagen. Pero resulta difícil que el sistema por sí mismo señale o diferencie entre la existencia o no de intención por parte del atacante en tener participación en la jugada, por lo cual es necesario remarcar que este dispositivo es únicamente un asistente y no un sustituto del árbitro[9].

A su vez, es importante señalar que el sistema descrito en este documento no determina en tiempo real el fuera de juego, sino que realiza la captura en un instante determinado para comenzar el análisis a partir de la imagen seleccionada y a petición de alguna autoridad, como se hace en otros deportes como el tenis[5], tenis de mesa[8], béisbol o fútbol americano. El único sistema que detecta en tiempo real, es la marcación del gol en el Hockey sobre hielo, ya que la velocidad del disco o puck alcanza los $160 \mathrm{~km} / \mathrm{h}[4,6]$.

\section{Entorno del proyecto}

\subsection{Regla del Fuera de Juego}

Para la realización del algoritmo presentado en este artículo es necesario conocer la regla del fuera de juego la cual es resultado de la reforma de 1960 en el reglamento de la FIFA donde se contempla en el punto 11 la regla del fuera de juego. En esta regla se indica que existe una posición de fuera de juego si un jugador se encuentra más cerca de la línea de meta (portería) contraria que el 
balón y el penúltimo adversario. Esto se toma en cuenta a partir del momento en que el balón es tocado o jugado por uno de sus compañeros con el fin de realizar un ataque[2]. Un ejemplo de esto se muestra en la Figura 2.

A su vez no existe posición de fuera de juego si el jugador está en su propia mitad de campo o a la misma altura que el penúltimo o los dos últimos adversarios (Figura 3). A su vez no hay infracción si un jugador recibe el balón en un saque de banda, un saque de meta o como resultado de un tiro de esquina[7].


Fig. 3. Ejemplo de posición reglamentaria en media cancha[2].

Dentro de la regla del fuera de lugar existe el término de juego activo el cual se presenta cuando un jugador se encuentre interfiriendo a un adversario o cuando haya ganado ventaja de la posición en la que se encuentra[10]. Por parte de la IFAB se toman algunas consideraciones para sancionar el fuera de juego; la primera de ellas plantea que se debe tener en consideración cualquier parte de la cabeza, cuerpo o pies del atacante en relación al penúltimo adversario, el balón o la línea media. Mientras que, para la interpretación de esta decisión, los brazos no se consideraran parte del cuerpo[1]. Dentro de la participación en el juego activo se toma en consideración que un jugador no está cometiendo una infracción simplemente por estar en posición de fuera de juego. Mientras que, existe infracción cuando existe posición de fuera de juego con la participación activa en el juego. Como consecuencia de la infracción por fuera de juego, el árbitro otorga un tiro libre indirecto que es lanzado desde el lugar donde estaba el jugador infractor en el momento en que el balón fue jugado o tocado por uno de sus compañeros. Dentro de esta regla se recomienda a los árbitros asistentes, quienes son los encargados de marcar esta regla es necesario que el árbitro se encuentre concentrado y centrar su atención, esto resulta clave en la determinación y sanción de la regla, también es importante considerar la colocación del árbitro para reducir el error en la marcación[2].

\section{Algoritmo del asistente para la determinación del fuera de juego}

El algoritmo del asistente para la determinación del fuera de juego parte de una imagen capturada mediante el uso de un sensor, Figura 4. Este algoritmo 
Asistente computarizado para la determinación de la regla del fuera de lugar en el fútbol soccer

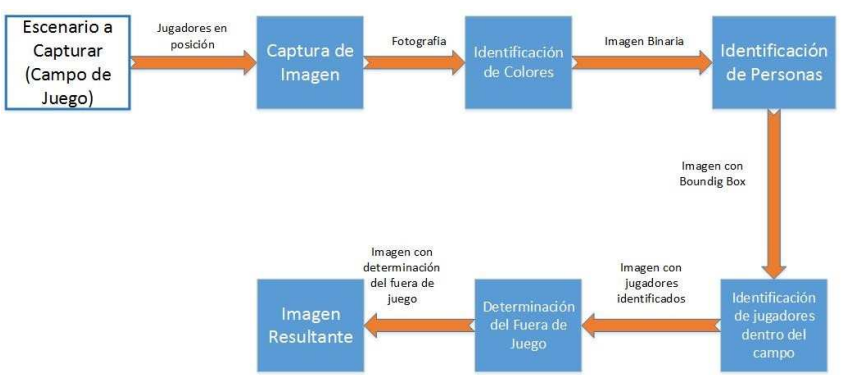

Fig. 4. Algoritmo del asistente para la determinación del fuera de juego.

busca que a partir de una imagen capturada se identifique el color del uniforme de ambos equipos para posteriormente identificar a cada jugador que se encuentre en el campo de juego. Así, el algoritmo ubica al ofensor más adelantado, así como al último defensor. Una vez hecho esto se compara la posición del ofensor contra el defensor y se determina si existe o no fuera de lugar.

\subsection{Captura de imagen}

El primer paso del algoritmo se realiza a partir de contar con un sensor compatible con el software MatLab, así como de contar con los elementos que se analizan dentro de un espacio de iluminación controlada, una vez que se cuenta con esto es posible realizar la captura de la imagen mediante la creación de un objeto de sistema el cual contiene las características del sensor conectado al dispositivo de cómputo que contenga el software MatLab R2014 o posterior.

\subsection{Identificación de colores}

Una vez que se tiene la imagen capturada, el siguiente paso es identificar el color principal de los uniformes de ambos equipos para con esto obtener una imagen que sólo muestre las áreas de interés, esto se realiza mediante un proceso de segmentación del color, el cual sigue los siguientes pasos:

1. Se toma un pixel dentro de la imagen $I$ con el color a identificar.

2. Se toma un valor de tolerancia el cual determina el intervalo de error $\varepsilon$.

3. Los resultados son almacenados dentro de un arreglo para los canales $I_{R}, I_{G}$ y $I_{B}$ con lo cual se calculan los valores de referencia.

4. Se realiza una comparación canal por canal con base en los valores de referencia y a la tolerancia señalada.

5. Mediante operaciones lógicas se comparan los resultados obtenidos.

6. Se realiza una segementación canal por canal para crear una imagen resultante $\widehat{I}$.

7. Se obtiene la imagen binaria $\bar{I}$ mediante la multiplicación de la imagen capturada por la imagen segmentada, es decir, $\bar{I}=I \times \widehat{I}$. 
Este análisis se realiza para los jugadores de ambos equipos, por lo cual se obtienen dos imágenes resultantes, $\bar{I}$ y su complemento $1-\bar{I}$, que representan las regiones con mayor cantidad de los colores indicados por el usuario al inicio de esta etapa, tomando en consideración la tolerancia registrada $\varepsilon$.

\subsection{Detección de personas}

Una vez obtenidas las imágenes binarias con los colores del uniforme de ambos equipos se utiliza esa información para conocer la posición de los jugadores dentro del terreno de juego, para esto se toma en cuenta la información proporcionada por las imágenes binarias antes generadas, $\bar{I}$ y $1-\bar{I}$. Así se obtiene una nueva imagen, la cual a partir de la imagen capturada original se muestra a cada uno de los elementos que se encuentren en el terreno de juego. Este proceso se realiza de la siguiente manera:

1. Se generan los objetos de sistema necesarios para habilitar las funciones de detección de personas.

2. Se establecen los parámetros necesarios para realizar la detección de personas.

3. Se realiza la detección de personas en cada una de las imágenes binarias obtenidas en la identificación de colores, $\bar{I}$ y $1-\bar{I}$.

4. Se crea la imagen resultante $I_{s}$ en la cual ya se encuentran señalados los elementos considerados persona dentro del terreno de juego.

Una vez terminado este proceso la imagen obtenida muestran a todos los jugadores de ambos equipos.

\subsection{Determinación del fuera de juego}

Una vez identificados todos los elementos necesarios para la detección del fuera de juego, el siguiente paso es realizar la determinación de la regla del fuera de juego, esto se realiza de la siguiente manera:

1. Una vez que se tienen todos los elementos detectados se identifica la ubicación en el eje x de cada uno de los jugadores utilizando los datos obtenidos en la detección de personas. Esto es posible mediante el uso de la información proporcionada por la etapa de detección de personas, donde a través de conocer la posición de los elementos detectados se ubican los dos más adelantados para ambos equipos los cuales son comparados entre sí.

2. Con la posición de ambos jugadores conocida se compara si la posición del atacante es menor a la posición del defensor, si esto ocurre no existe fuera de juego en caso contrario se determina el fuera de juego. Es por ello que se consideran los dos valores en el eje de las abscisas de los jugadores más adelantados mediante el uso de una sustracción Pos $=X_{J_{1}}-X_{J_{2}}$ de la siguiente manera:

- Primeramente, si el valor de la sustracción Pos $>0$ no existe fuera de juego. 
- En caso contrario si el valor de la sustracción Pos $\leq 0$ existe fuera de juego.

3. Una vez que comparadas las posiciones de ambos jugadores y se determina el fuera de juego se crea una nueva imagen resultante $I_{\text {offside }}$ la cual indica la existencia o no del fuera de juego. Un ejemplo del resultado $I_{\text {offside }}$ de aplicar este algoritmo propuesto se observa en las Figuras 5 y 6 .

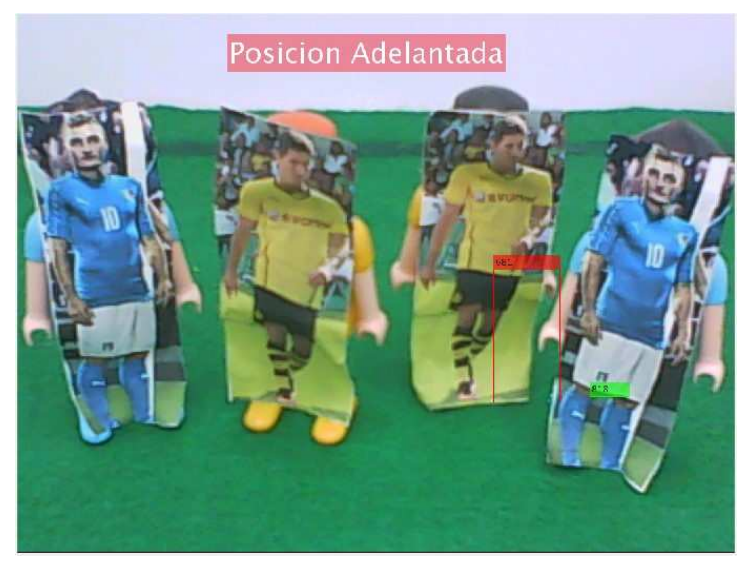

Fig. 5. Resultado del algoritmo propuesto o $I_{\text {offside, }}$ se detecta que hay Posición Adelantada.

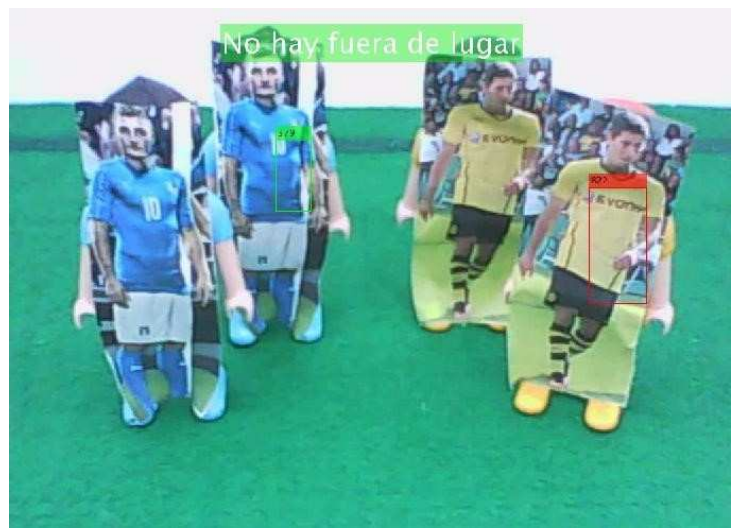

Fig. 6. Resultado del algoritmo propuesto o $I_{\text {offside, }}$, se detecta que no hay Fuera de Lugar.

\section{Resultados experimentales}

Una vez realizadas cada una de las etapas del algoritmo lo siguiente es conocer si el algoritmo propuesto es capaz de determinar el fuera de juego, 
así como las condiciones necesarias para determinarlo. Por lo cual, mediante una serie de capturas utilizando figuras Playmobil@ a las cuales se les pegan imágenes de jugadores de fútbol con distintos colores de uniforme, siendo el equipo azul el equipo ofensivo y el equipo amarillo el defensivo, con lo cual se ha considerado que el descriptor de HOG utilizado en la fase de detección de personas se encuentra más familiarizado con aquellas figuras que asemejen la estructura del ser humano.

Otro punto importante que se considera son aquellos casos donde al acercar los jugadores de ambos equipos de forma que no sean perceptibles totalmente por el sensor o incluso en casos donde el sensor no es capaz de detectarlos lo cual produce que sólo aquellos jugadores detectados por la cámara sean considerados para el análisis del asistente para la determinación del fuera de juego, a estas situaciones se les llama casos críticos.

El rango del sensor utilizada para el experimento es de $90^{\circ}$ con respecto al terreno de juego, mientras que la altura está determinada por las características físicas del sensor. Así, se presentan dos casos para evaluar el funcionamiento del algoritmo los cuales permiten diferenciar la existencia o no del fuera de juego los cuales son:

1. Consiste en tener al equipo ofensor en posición reglamentaria

2. Un atacante fue desplazado por delante de la línea defensiva con lo cual se espera que el algoritmo determine la posición del fuera de juego.

Para este experimento se determinaron los siguientes parámetros dentro del código del algoritmo:

1. Rango de radio del balón: 71 a 78 pixeles

2. Polaridad de Balón: Clara

3. Sensibilidad de detección del balón: 0.9822

4. Umbral de detección: 0.26

5. Factor de escala para personas: 1.7222

6. Tamaño mínimo: 102 a 49 pixeles

Así, se propone la modificación de la altura del sensor para una mejor captura, así como establecer un ambiente controlado donde el brillo y la sombra percibida en el terreno de juego disminuyan. La altura del sensor se modifica a $18 \mathrm{~cm}$ sobre el terreno de juego y con respecto al algoritmo se realizaron los siguientes cambios:

1. El rango de radio cambia de entre 50 y 55 pixeles.

2. Se mantiene la polaridad del balón en clara.

3. Sensibilidad de detección del balón: 0.98225

4. Umbral de detección a un valor de 0.2655

5. Para este caso, se descarta el factor de escala para personas: 1.7222

6. De igual manera, se descarta el tamaño mínimo: 102 a 49

7. Mismo modelo de detección

8. Umbral de clasificación de personas: 1.65 
Una vez que se realizaron estas modificaciones se capturan en las mismas posiciones que se utilizaron en el caso anterior. En este experimento se demuestra que es posible determinar el fuera de juego mediante el uso del algoritmo propuesto, tomando en consideración que los pixeles seleccionados proporcionen la información suficiente para la detección de colores y personas, mientras que la modificación del Ángulo facilita la detección del balón, lo cual permite pasar al algoritmo de fase de determinación del fuera de juego.

Un problema encntrado al aplicar el algoritmo en este segundo modelo es que en las capturas que no se detecta el fuera de juego se observa que la función peopleDetector determina el orden de las personas considerando la distancia focal del sensor. Esto provoca que al solicitar al elemento que se encuentre más adelantado se detecta al elemento más cercano al sensor.

Al modificar la posición del sensor y tomar en cuenta la función peopleDetector que toma en cuenta la distancia focal al propio sensor, ahora es necesario tomar en consideración la posición de los elementos en el eje de las abscisas, con lo que es posible obtener los resultados almacenados en el arreglo bboxes de la función step de la biblioteca peopleDetector donde la primera columna contiene los valores de $\mathrm{x}$ en el eje de las abscisas.

Una vez que se obtienen los valores en el arreglo se hacn las siguientes modificaciones al algoritmo:

1. Se extraen los valores de $\mathrm{x}$ del arreglo bboxes, los valores se almacenan en un nuevo arreglo.

2. Se busca el valor máximo dentro del arreglo, que es el valor más grande dentro del eje x el cual representa al jugador más alejado del extremo izquierdo de la imagen.

3. Una vez que se encuentro el valor más grande se busca su posición dentro del arreglo bboxes.

4. Se buscó el valor asociado a la fila de valor x para obtener las coordenadas en el eje de las ordenadas.

5. Se realizan los pasos del 2 al 4 para el arreglo bboxes2 y se obtienen los puntos $x_{2}$ y $y_{2}$

6. Conocidos los puntos $x_{2}$ y $y_{2}$ y al aplicar la ecuación de la recta se obtiene la recta entre los dos puntos.

7. Se obtuvo el punto medio de la recta mediante la ecuación $x_{m}=\frac{x_{2}+x_{1}}{2}$

8. Se compara el punto medio de la imagen y el punto medio de la recta, lo cual se aplica bajo las siguientes condiciones:

a) Si el valor del punto medio de la resta es mayor que el obtenido en la imagen se realiza lo siguiente:

1) Se realiza una sustracción entre el valor de ambos puntos.

2) El resultado de esta operación es el número de columnas dentro de la imagen que se eliminan.

3) Se crea un arreglo que contiene la última columna de la imagen

4) Se realiza la concatenación de imágenes, así en primer lugar se inserta la imagen sustraída de la original y posteriormente el arreglo repetido la cantidad de columnas que se eliminaron en la imagen original. 
b) Si el valor del punto de la recta es menor que el obtenido en la imagen se realiza los siguiente:

1) El resultado de esta operación es el número de columnas máximo que tiene la imagen

2) Se crea un arreglo que contiene la primera columna de la imagen.

3) Se realiza la concatenación de imágenes primeramente se inserta en el arreglo repetido de la cantidad de columnas que se eliminan en la imagen original y posteriormente la imagen sustraída de la original.

c) En caso de que ambos puntos se encuentren en la misma posición no se modifican en la imagen.

9. Para determinar el fuera de juego se realiza una sustracción entre los puntos $x_{1}$ y $x_{2}$ obtenidos del arreglo bboxes por lo que se considera lo siguiente:

a) Si el valor de la sustracción es mayor que cero no existe fuera de lugar.

b) Si el valor de la sustracción es menor o igual que cero existe fuera de lugar.

Al considerar que el porcentaje obtenido en el experimento anterior no es lo suficientemente alto para considerar que el objetivo del asistente para la determinación del fuera de juego se cumple. Ahora, se considera que la forma de las figuras Playmobil no representan con exactitud a un ser humano, lo cual provoca que la función peopleDetector encuentre a todos los jugadores en el terreno de juego.

Para solucionar esto se utilizan fotos de jugadores de futbol con los mismos colores de uniforme que las figuras playmobil sobre estas y con ello se realizan pruebas en diferentes posiciones del terreno de juego tanto en posición reglamentaria como en fuera de juego.

A su vez se consideran algunos casos críticos los cuales se presentan al acerca los jugadores de ambos equipos de forma que no son perceptibles totalmente por el sensor o incluso en casos donde el sensor no es capaz de detectarlos, lo cual produce que sólo aquellos jugadores detectados por el sensor sean considerados para el análisis del asistente para la determinación del fuera de juego.

Una vez que el asistente demostró su funcionamiento, resulta necesario conocer su tiempo de respuesta, para lo cual se realizaron 30 tomas, 15 en posición reglamentaria con 5 casos críticos y 15 en posición de fuera de juego con 5 casos críticos. Obteniendo los resultados mostrados en las Tablas 1 y 2.

De los resultados obtenidos se concluye que de las 15 en posición reglamentaria con sus 5 casos críticos, 12 resultaron aciertos con 4 casos críticos positivos, lo que esto representa un $80 \%$ de efectividad con un tiempo promedio de 72.98 ms, mientras que para las 15 posiciones de fuera de juego con sus 5 casos críticos, 11 resultaron positivos con 2 casos críticos positivos lo que representa un $73.3 \%$ de efectividad con un tiempo promedio de $143.53 \mathrm{~ms}$.

De los resultados obtenidos se concluye que de las 30 capturas en posición reglamentaria, 27 de ellas resultaron correctas, lo que representa un porcentaje de efectividad del $90 \%$. Mientras que de las 30 capturas en posición de fuera de juego 23 resultaron correctas lo que representa un $73.67 \%$ de efectividad. Por lo tanto, contabilizando las 60 pruebas totales resultando correctas 52 capturas 
Asistente computarizado para la determinación de la regla del fuera de lugar en el fútbol soccer

Tabla 1. Resultados obtenidos en el Experimento 4 en Posición Reglamentaria.

\begin{tabular}{|c|c|c|c|}
\hline Muestra & Caso Crítico & Detección & Tiempo $(\mathrm{ms})$ \\
\hline 1 & No & Sí & 166.136 \\
\hline 2 & No & Sí & 23.69 \\
\hline 3 & No & Sí & 19.59 \\
\hline 4 & No & No & 17.829 \\
\hline 5 & No & Sí & 16.86 \\
\hline 6 & No & No & 18.16 \\
\hline 7 & No & $\mathrm{Si}$ & 17.82 \\
\hline 8 & No & $\mathrm{Si}$ & 19.059 \\
\hline 9 & No & $\mathrm{Si}$ & 21.32 \\
\hline 10 & No & $\mathrm{Si}$ & 19.13 \\
\hline 11 & Sí & $\mathrm{Si}$ & 75.72 \\
\hline 12 & Sí & $\mathrm{Si}$ & 297.12 \\
\hline 13 & Sí & No & 304.1 \\
\hline 14 & Sí & Sí & 48.83 \\
\hline 15 & Sí & Sí & 29.39 \\
\hline
\end{tabular}

Tabla 2. Resultados obtenidos en el Experimento 4 en posición de fuera de juego.

\begin{tabular}{|c|c|c|c|}
\hline Muestra & Caso Crítico & Detección & Tiempo $(\mathrm{ms})$ \\
\hline 1 & Sí & No & 27.17 \\
\hline 2 & Sí & Sí & 24.37 \\
\hline 3 & Sí & No & 31.18 \\
\hline 4 & Sí & No & 454.19 \\
\hline 5 & Sí & $\mathrm{Si}$ & 60.52 \\
\hline 6 & No & $\mathrm{Si}$ & 29.21 \\
\hline 7 & No & $\mathrm{Si}$ & 22.73 \\
\hline 8 & No & $\mathrm{Si}$ & 23.35 \\
\hline 9 & No & No & 20.85 \\
\hline 10 & No & $\mathrm{Si}$ & 42.17 \\
\hline 11 & No & $\mathrm{Si}$ & 282.33 \\
\hline 12 & No & $\mathrm{Si}$ & 120.48 \\
\hline 13 & No & SÍ & 524.3 \\
\hline 14 & No & Sí & 450.77 \\
\hline 15 & No & Sí & 39.33 \\
\hline
\end{tabular}

se obtiene un porcentaje del $86.6 \%$ de efectividad total del asistente para la determinación del fuera de juego.

\section{Conclusiones}

Finalmente, como resultado de los experimentos realizados se denuestra que es posible desarrollar un asistente para la detección del fuera de juego por medio de Visión por computadora utilizando procesos de segmentación y detección de objetos utilizando la herramienta MatLab.

A su vez se concluye que para que el objetivo de la determinación del fuera de juego tenga el menor error posible es necesario contar con las condiciones físicas ideales como son una iluminación uniforme dentro del terreno de juego, además de considerar que los elementos que se encuentren dentro de este se asemejan lo más posible a la forma del ser humano, esto con el fin de evitar falsos positivos y errores que no permitan la detección del fuera de juego. También se demuestra que es posible desarrollar un asistente para la determinación del fuera 
de juego en un tiempo aceptable siempre y cuando las condiciones del equipo de procesamiento lo permitan, ya que de no ser así esto retrasa la respuesta, lo cual no resuleve la problemática en el análisis de esta regla en el campo de juego.

Agradecimientos. Este trabajo es desarrollado con recursos e instalaciones del Instituto Politécnico Nacional, México, por medio del Proyecto SIP 20180514 y la Comisión de Operación y Fomento de Actividades Académicas (COFAA). Cabe resaltar que este trabajo es parte de la tesis de Nivel Licenciatura del Becario BEIFI Joshua Romero Tapia, dirigida por el Dr. Jesús Jaime Moreno Escobar. También, se le agradece por un lado a la Ing. Isabel Meraz por el apoyo soporte lógistico y técnico; y por otro a los revisores que aportaron sus valiosos conocimientos para mejora del presente artículo.

\section{Referencias}

1. Aribowo, A., Gunawan, G., Tjahyadi, H.: Adaptive edge detection and histogram color segmentation for centralized vision of soccer robot. In: 2016 International Conference on Informatics and Computing (ICIC). pp. 49-54 (Oct 2016)

2. FIFA: Reglas de Juego 2015/2016. FIFA, FIFA-Strasse 20 Apdo. postal 8044 Zúrich Suiza (2015)

3. Gholami, A., Bigham, B.S.: A learned soccer goalkeeper petri net model. In: 2017 Artificial Intelligence and Robotics (IRANOPEN). pp. 102-108 (April 2017)

4. Hardegger, M., Ledergerber, B., Mutter, S., Vogt, C., Seiter, J., Calatroni, A., Tröster, G.: Sensor technology for ice hockey and skating. In: 2015 IEEE 12th International Conference on Wearable and Implantable Body Sensor Networks (BSN). pp. 1-6 (June 2015)

5. Leong, L.H., Zulkifley, M.A., Hussain, A.B.: Computer vision approach to automatic linesman. In: 2014 IEEE 10th International Colloquium on Signal Processing and its Applications. pp. 212-215 (March 2014)

6. Pileggi, H., Stolper, C.D., Boyle, J.M., Stasko, J.T.: Snapshot: Visualization to propel ice hockey analytics. IEEE Transactions on Visualization and Computer Graphics 18(12), 2819-2828 (Dec 2012)

7. Song, X., Zhou, Z., Guo, H., Zhao, X., Zhang, H.: Adaptive retinex algorithm based on genetic algorithm and human visual system. In: 2016 8th International Conference on Intelligent Human-Machine Systems and Cybernetics (IHMSC). vol. 01, pp. 183-186 (Aug 2016)

8. Wong, P.K.C.: Developing an intelligent assistant for table tennis umpires. In: First Asia International Conference on Modelling Simulation (AMS'07). pp. 340-345 (March 2007)

9. Yao, Q., Kubota, A., Kawakita, K., Nonaka, K., Sankoh, H., Naito, S.: Fast camera self-calibration for synthesizing free viewpoint soccer video. In: 2017 IEEE International Conference on Acoustics, Speech and Signal Processing (ICASSP). pp. 1612-1616 (March 2017)

10. Yu, X., Leong, H.W., Xu, C., Tian, Q.: Trajectory-based ball detection and tracking in broadcast soccer video. IEEE Transactions on Multimedia 8, 1164-1178 (2006) 